\title{
Modulation of smoke-induced DNA and microRNA alterations in mouse lung by licofelone, a triple COX-1, COX-2 and 5-LOX inhibitor
}

\author{
Alberto Izzotti ${ }^{1,2}$, Roumen Balansky ${ }^{3}$, Rosanna T.Micale ${ }^{1}$, Alessandra Pulliero ${ }^{1}$, \\ Sebastiano La Maestra ${ }^{1}$ and Silvio De Flora ${ }^{1, *}$
}

${ }^{1}$ Department of Health Sciences, University of Genoa, 16139 Genoa, Italy, ${ }^{2}$ RCCS Policlinico San Martino, Genoa, Italy and ${ }^{3}$ National Center of Oncology, Sofia 1756, Bulgaria

*To whom correspondence should be addressed. Tel: +39 010 3538500; Fax: +39 010 3538482; Email: sdf@unige.it

\begin{abstract}
Chronic inflammation plays a crucial role in the carcinogenesis process and, in particular, in smoking-related carcinogenesis. Therefore, anti-inflammatory agents provide an interesting perspective in the prevention of smokingassociated cancers. Among nonsteroidal anti-inflammatory drugs (NSAIDs), licofelone is a triple inhibitor of both cyclooxygenases (COX-1 and COX-2) and of 5-lipooxygenase (5-LOX) that has shown some encouraging results in cancer prevention models. We previously showed that the dietary administration of licofelone, starting after weanling, to Swiss $\mathrm{H}$ mice exposed for 4 months to mainstream cigarette smoke since birth attenuated preneoplastic lesions of inflammatory nature in both lung and urinary tract, and had some effects on the yield of lung tumors at 7.5 months of age. The present study aimed at evaluating the early modulation by licofelone of pulmonary DNA and RNA alterations either in smoke-free or smoke-exposed $\mathrm{H}$ mice after 10 weeks of exposure. Licofelone protected the mice from the smoke-induced loss of body weight and significantly attenuated smoke-induced nucleotide alterations by decreasing the levels of bulky DNA adducts and 8-hydroxy-2'-deoxyguanosine in mouse lung. Moreover, the drug counteracted dysregulation by smoke of several pulmonary microRNAs involved in stress response, inflammation, apoptosis, and oncogene suppression. However, even in smoke-free mice administration of the drug had significant effects on a broad panel of microRNAs and, as assessed in a subset of mice used in a parallel cancer chemoprevention study, licofelone even enhanced the smoke-induced systemic genotoxic damage after 4 months of exposure. Therefore, caution should be paid when administering licofelone to smokers for long periods.
\end{abstract}

\section{Introduction}

Inhalation of mainstream cigarette smoke (MCS) is causally associated with cancers affecting several anatomical sites in humans (1) and plays an important role in the causation of a variety of other chronic degenerative diseases, such as chronic obstructive pulmonary diseases, cardiovascular diseases, and cerebrovascular diseases (2). The most obvious strategy to prevent smoking-related diseases is to avoid exposure to MCS either by refraining from smoking (never-smokers) or by quitting smoking (ex-smokers). This strategy has already proven to attenuate the epidemic of lung cancer either in selected groups of population or in the whole male population of several countries in which a decrease in the consumption of cigarettes has occurred (3). As a complementary approach, chemoprevention by means of dietary and pharmacological agents may render the organism more resistant to the attack of the multiple carcinogens contained in MCS and to interfere with carcinogenesis via a large variety of mechanisms (4). Besides being addressed to passive smokers, who are exposed to environmental cigarette smoke (ECS), chemoprevention targets those active smokers who are addicted to nicotine and are unable to quit smoking. 


\begin{tabular}{ll} 
Abbreviations: & \\
\hline AA & arachidonic acid; \\
COX & cyclooxygenase; \\
CYP & cytochrome P450; \\
DFMO & difluoromethylornithine; \\
DRZ & diagonal radioactive zone; \\
ECS & environmental cigarette smoke; \\
EGFR & epidermal growth factor receptor; \\
HCA & hierarchical cluster analysis; \\
LTE & leukotriene; \\
LOX & lipooxygenase; \\
MCS & mainstream cigarette smoke \\
miRNA & microRNA; \\
MN NCE & micronucleated normochromatic \\
& erythrocytes; \\
NNK & 4-(methylnitrosamino)-1-(3-pyridyl)- \\
& 1-butanone; \\
NSAIDs & nonsteroidal anti-inflammatory \\
& drugs; \\
8-oxodGuo & 8-hydroxy-2'-deoxyguanosine; \\
PCA & principal component analysis; \\
PGE & prostaglandin endoperoxide; \\
SPA & scatter-plot analysis; \\
VPA & volcano-plot analysis \\
\hline &
\end{tabular}

A further target is represented by ex-smokers, constituting a broad proportion of the population, who for several years have an increased risk to develop lung cancer compared with neversmokers. To give an idea of the importance of chemoprevention in lung cancer prevention, it has been estimated that about 234,000 new cases of lung cancer will be diagnosed in the USA in 2018 (5). Since the approximate latency time for lung cancer has been estimated to be 30 years (6), and even assuming a further decline of lung cancer incidence in next years, this means that the disease has already started its course in about 7 million people, in whom lung cancer will be diagnosed from today to 2049. It is a huge population in which any effort should be made to inhibit or to delay the growth of the neoplastic mass.

Chronic inflammation plays a crucial role among the mechanisms involved in the carcinogenesis process and, in particular, in smoking-related carcinogenesis (7-9). Accordingly, anti-inflammatory agents provide an interesting perspective in the prevention of smoking-associated cancers (10). In this context, the main mechanism of nonsteroidal anti-inflammatory drugs (NSAIDs), which are among the most widely used drugs worldwide, is to interfere with the metabolism of arachidonic acid (AA), a polyunsaturated omega- 6 essential fatty acid $(\omega-6)$ that is the substrate for different enzyme systems. These include cytochromes P450 (CYP), lipoxygenases (LOX), and cyclooxygenases (COX), which catalyze arachidonic acid oxygenation thereby generating prostaglandin endoperoxides (PGE). In addition, NSAIDs may reduce the 'inflammogenesis of cancer' by lowering the levels of inflammation mediators, such as cytokines, chemochines, and adhesion molecules that are involved in airway and pulmonary diseases (11-13). While COX-1 is the housekeeping isoform, the PGEs derived from COX-1 being involved in the homeostatic maintenance of the gastric mucosa, COX-2 is the inducible isoform, which is expressed in response to certain stimuli such as mitogens, cytokines and growth factors having a pro-inflammatory function (14). Arachidonic acid is metabolized by 5-LOX to leukotrienes (LTEs), one of which (LTE-B4) has been shown to be involved in the development of gastrointestinal ulcers (15). Increased levels of urinary PGE-M and LTE (4) were found in human smokers, a result that may reflect subclinical lung inflammation, and administration of the selective COX-2 inhibitor celecoxib shunted arachidonic acid into the proinflammatory 5-LOX pathway. Since 5-LOX activity and LTE (4) play a role in cardiovascular disease, these results may help to explain the link between use of COX-2 inhibitors and cardiovascular complications (16). Thus, targeting COX and 5-LOX pathways may provide better efficacy without unwanted cardiovascular and gastrointestinal side effects (17).

Using a murine model of MCS-related carcinogenesis, we previously investigated the ability of a variety of pharmacological agents to modulate the yield of lung tumors and other histopathological alterations [see a review in ref. (18)]. Among anti-inflammatory drugs, we investigated the cancerprevention ability of 4 NSAIDs, including the selective COX-2 inhibitor celecoxib (19), the COX-1 and COX-2 inhibitors aspirin and naproxen (20), and the triple COX-1, COX-2, and 5-LOX inhibitor licofelone (19). In addition, we evaluated the properties of celecoxib (21), naproxen and aspirin (22) to modulate MCSrelated alterations of genomic and epigenetic nature in mice.

Licofelone (2-[6-(4-chlorophenyl)2,2-dimethyl-7-phenyl2,3-dihydro-1H-pyrrolizin-5-yl]acetic acid, CAS 156897-06-2), a triple inhibitor of both COXs and 5-LOX, has been proposed as a new tool in anti-inflammatory therapy (23), particularly in the treatment of osteoarthritis (24). In addition, both COXs and 5-LOX have been implicated in carcinogenesis and especially in colon carcinogenesis, and the striking interrelationship of their biological functions suggests that molecules that are able to block both COX-2 and 5-LOX pathways may provide a promising approach to colon cancer prevention (17). Using experimental test systems, licofelone was found to inhibit the formation of tumors, such as spontaneous intestinal tumors in $\mathrm{APC}^{\mathrm{Min} /+}$ mice $(17,25)$ and spontaneous urothelial cell carcinoma growth and invasion in UPII-SV40T transgenic mice (26), squamous cell carcinomas of the tongue in male F344 rats receiving 4-nitroquinoline-1-oxide in the drinking water (27), and lung adenomas induced by benzo[a]pyrene in female A/J mice receiving licofelone via oropharyngeal aspiration (28). In addition, especially when combined with the epidermal growth factor receptor (EGFR) inhibitor gefinitib, licofelone inhibited the incidence of pancreatic intraepithelial neoplasms and their progression to pancreatic ductal adenocarcinoma using p48Cre/tLSL-Kras ${ }^{\mathrm{G} 12 \mathrm{D} /+}$ mice expressing the activated $\mathrm{Kras}^{\mathrm{G} 12 \mathrm{D}}$ oncogene (29). In that model, miRNAs characteristics of pancreatic tumorinitiating/cancer stem cells and inflammation were modulated by this NSAID (30). Licofelone also inhibited the formation of 4-(methylnitrosamino)-1-(3-pyridyl)-1-butanone (NNK)-induced lung tumors in $\mathrm{A} / \mathrm{J}$ mice, an effect that was potentiated by the simultaneous administration of difluoromethylornithine (DFMO) (31).

We previously demonstrated that the dietary administration of licofelone, starting after weanling, to Swiss $\mathrm{H}$ mice of both genders exposed for 4 months to MCS since birth attenuated preneoplastic lesions of inflammatory nature in both lung and urinary tract, and had some effects on the yield of lung tumors at 7.5 months of age (19). We report herein the results of an independent study in which we evaluated, under the same experimental conditions, the early modulation by licofelone of pulmonary DNA and RNA alterations either in smoke-free or MCS-exposed mice after 10 weeks of exposure. Nucleotide alterations were investigated by measuring bulky DNA adducts and 8-hydroxy-2'-deoxyguanosine (8-oxodGuo), and RNA alterations were evaluated by determining the expression of 1,135 mouse microRNAs (miRNAs). The results obtained show that licofelone is able to significantly attenuate MCS-induced DNA alterations 
in mouse lung and to counteract dysregulation by MCS of several miRNAs. However, in smoke-free mice, administration of the drug had significant effects on an even broader panel of miRNAs and, as assessed in a subset of mice used in the cancer chemoprevention study (19), licofelone even enhanced the MCSinduced systemic genotoxic damage after 4 months of exposure.

\section{Materials and methods}

\section{Mice}

Strain $\mathrm{H}$ mice, originated from Swiss albino mice, were used. These mice are sensitive to the induction of preneoplastic and neoplastic lung alterations induced by MCS (32). The mice were originated and bred in the Animal Laboratory of the National Center of Oncology (Sofia, Bulgaria) and shipped to the Department of Health Sciences at the University of Genoa (Italy), where they were housed in Makrolon ${ }^{\mathrm{TM}}$ cages, maintained on standard rodent chow (Teklad 2018, Harlan Laboratories, San Pietro al Natisone, Udine, Italy), and given drinking water ad libitum. The animal room temperature was $23 \pm 2^{\circ} \mathrm{C}$, with a relative humidity of $55 \%$ and a $12 \mathrm{~h}$ day-night cycle. Housing and treatments of mice were in accordance with European (2010/63 UE Directive) and institutional guidelines. The issuance of the Office of Laboratory Animal Welfare (OLAW) with the University of Genoa bears the identification number A5899-01 and is effective until 28 February 2021.

\section{Exposure to MCS}

A whole-body exposure of mice to MCS was carried out as previously described (32), by using 3R4F Kentucky reference cigarettes (College of Agriculture, The Reference Cigarette Program, University of Kentucky, Lexington, KY, USA), having a declared content of $9.4 \mathrm{mg}$ tar and $0.73 \mathrm{mg}$ nicotine, and delivering $12 \mathrm{mg}$ of CO each when burned. MCS was delivered to the exposure chambers by drawing 15 consecutive puffs, each of $60 \mathrm{ml}$ and lasting $6 \mathrm{~s}$. Each daily session involved 6 consecutive exposures, lasting $10 \mathrm{~min}$ each, with 1 min intervals during which a total air change was made in order to avoid sufferance of mice. Exposure started within $12 \mathrm{~h}$ after birth and continued daily until the end of the experiment (10 weeks). The average total particulate matter in the exposure chambers was $684 \mathrm{mg} / \mathrm{m}^{3}$.

\section{Licofelone}

Licofelone was supplied by the US NCI via MRIGlobal (Kansas City, MO, USA). As previously reported (19), a subchronic toxicity study was carried out in $\mathrm{H}$ mice by incorporating the drug in the diet at the doses of 150, 300,600 and $1200 \mathrm{mg} / \mathrm{kg}$. These doses were selected based on literature data relative to experimental studies in mice and by taking into account the therapeutic doses used in humans. At any licofelone dose, neither sufferance nor alterations in the behavior of mice were detected at daily inspections nor the body weight gain was affected by more than $10 \%$ as compared with controls. Therefore, the $80 \%$ of the highest tested dose, corresponding to $960 \mathrm{mg}$ licofelone/kg diet, was used in the chemoprevention study.

\section{Chemoprevention study}

This study used a total of 40 neonatal mice, which were divided into the following 4 groups, each composed of 5 males and 5 females: Group 1. Mice kept in filtered air (sham-exposed mice); Group 2. Mice receiving licofelone with the diet after weanling (licofelone-treated mice); Group 3. Mice exposed to MCS since birth (MCS-exposed mice); Group 4. Mice exposed to MCS since birth and receiving licofelone with the diet after weaning (MCSexposed mice treated with licofelone). At 10 weeks of age, i.e. 6 weeks after weaning, all mice were sacrificed and their lungs were collected. The right lung of each mouse was immersed in RNAlater ${ }^{\circledR}$ solution for $24 \mathrm{~h}$ and then transferred at $-80^{\circ} \mathrm{C}$ until RNA extraction and analysis. The left lung was directly transferred at $-80^{\circ} \mathrm{C}$ until DNA extraction and analysis.

\section{Extraction and analysis of lung DNA}

As previously described (33), DNA was extracted from tissue specimens $(50 \mathrm{mg}$ ) collected individually from the left lungs of 40 mice. The specimens were homogenized by using a tissue lyser (TissueLyser, Qiagen,, Gaithersburg, MD, USA) and, after centrifugation at $14000 \times g$ at $4^{\circ} \mathrm{C}$ for 15 min, DNA was purified from the supernatant by using a commercially available kit (GenElutt ${ }^{\top \mathrm{M}}$ Mammalian Genomic DNA Miniprep kit, Sigma, St Louis, MO, USA). Spectrophotometric analyses by means of a fiber optic spectrophotometer (Nanodrop ND-1000, Thermo Scientific, Wilmington, DE, USA) showed a satisfactory quality of the DNA extracted from the 40 processed samples, with an average $260 / 280$ ratio of $1.9 \pm 0.01$ (mean $\pm S E$ ). Bulky DNA adducts, after butanol enrichment, and 8-oxodGuo were measured by ${ }^{32} \mathrm{P}$ postlabeling procedures as previously described (34).

\section{Modulation of the systemic MCS genotoxicity}

Modulation by licofelone of the systemic MCS genotoxicity was evaluated in a subset of mice used in a parallel cancer chemoprevention study (19). At 4 months of age, immediately after discontinuing exposure to MCS, peripheral blood was collected from the tail lateral vein and smeared onto slides ( 2 slides/mouse) from 20 mice (10 males and 10 females) per each one of the experimental groups, including (a) sham-exposed mice, (b) MCS-exposed mice in the absence of the chemopreventive agent and (c) MCS-exposed mice receiving licofelone with the diet after weaning. After staining of blood smears with May-Grünwald-Giemsa, the frequency of micronucleated normochromatic erythrocytes (MN NCE) was scored by analyzing microscopically 50000 NCE/mouse.

\section{Extraction and analysis of lung RNA and expression of miRNAs}

For RNA extraction, fragments (10 mg each) of right lungs were homogenized in QIAzol Lysis Reagent by continuous shaking in Tissue Lyser (Qiagen Hilden, Germany). After centrifugation at $14000 \times g$ at $4^{\circ} \mathrm{C}$ for $15 \mathrm{~min}$, RNA was purified by using a commercially available kit (miRNeasy, Qiagen). Spectrophotometric analyses by fiber optic spectrophotometer (Nanodrop ND-1000) indicated a satisfactory quality of the RNA extracted from all samples, with an average $260 / 280$ ratio of $2.1 \pm 0.16$ (mean \pm SE). The RNA structural integrity was evaluated by capillary electrophoresis using a RNA bioanalyzer (Bioanalyzer Agilent 2100, Agilent Santa Clara, CA, USA) equipped with a RNA oligonucleotide chip (RNA 6000 Nano Ladder Chip, Agilent). The RNA Integrity Number (RIN), which is obtained by calculating the ratio between ribosomal RNA bands and total RNA, was $6.5 \pm 0.21$ $($ mean $\pm \mathrm{SE})$. The specimens collected from all experimental groups were processed as two pools composed of 5 mice each, one for males and the other one for females.

The expression of pulmonary miRNAs was evaluated by using the seventh-generation miRCURY LNA ${ }^{\mathrm{TM}}$ microRNA Array (Exiqon), which contains 3100 capture probes covering human, mouse and rat miRNAs. In particular, this array covers 1135 mouse miRNAs, which correspond to the $88.6 \%$ of the 1281 mouse miRNAs listed in miRBase 19. miRNA microarray expression data have been recorded in GEO database (GEO accession number requested).

For miR-30b, microarray results in the four experimental groups were validated by qPCR. The standard qPCR SYBR green procedure was used, as described elsewhere (22). Four replicates of each sample were analyzed.

\section{Statistical analysis}

The differences among the experimental groups relative to body weights, frequency of MN NCE, and levels of pulmonary DNA adducts and 8-oxodGuo, all of them expressed as means \pm SE within each group of mice, were evaluated for statistical significance by ANOVA followed by Student's test for unpaired data. miRNA microarray data were analyzed by GeneSpring software (Agilent, Santa Clara, CA, USA) after local background subtraction, log transformation and normalization, and expression data were median centered by using the GeneSpring normalization option. The overall treatment-related variability of microarray data was evaluated by scatter-plot analysis (SPA), hierarchical cluster analysis (HCA), sprincipal component analysis (PCA) and volcano-plot analysis (VPA). The statistical significance of the differences among experimental groups was evaluated by means of the GeneSpring ANOVA by using Bonferroni multiple testing correction. Differences between sets of data, inferred from volcano-plot analysis, were taken as significant when they showed at least a 2-fold variation and were statistically significant $(P<0.05)$. 


\section{Results}

Licofelone does not affect survival and protects the mice from the slight MCS-induced loss of body weight gain

All the 40 mice used for the evaluation of nucleotide modifications and microRNA expression survived throughout duration of the experiment. Supplementary Table S1, available at Carcinogenesis Online, shows the body weights of these mice, which were measured at weekly intervals after weaning until the end of the experiment. Exposure to MCS, starting soon after birth, resulted in a slight decrease of body weight gain in both males and females. Such a body weight loss was moderate but statistically significant in males from week 3 after weaning, i.e. approximately at 7 weeks of life, and in females from week 4 onwards, i.e. at 8 weeks of life. In any case, the maximum body weight loss in MCS-exposed mice, compared with shamexposed mice, was the $15.3 \%$ in males and the $7.9 \%$ in females. Administration of licofelone did not affect the body weight gain in smoke-free mice, whereas the drug exerted some protection in MCS-exposed mice. In fact, in MCS-exposed females treated with licofelone there was no significant difference, at any time, compared with sham-exposed females. In MCS-exposed males treated with licofelone there was a complete recovery after 6 weeks, when the body weight was significantly higher as compared with MCS-exposed mice in the absence of the drug.

\section{Licofelone inhibits the MCS-induced formation of bulky DNA adducts in lung}

Exposure of mice to MCS resulted, at ${ }^{32} \mathrm{P}$ postlabeling, in the formation of a diagonal radioactive zone (DRZ, Figure 1). Table 1 (left column) summarizes the results of the analysis of bulky DNA adducts in the lung of 40 mice. On an average, MCS increased DNA adduct levels 13.7-fold in males and 16.3-fold in females. Licofelone did not affect DNA adduct levels in smoke-free mice but significantly attenuated the increase induced by MCS both in males, with a 2.1-fold decrease, and in females, with a 2.0-fold decrease. Inhibition of MCS-related DNA adducts by licofelone mainly affected slowly migrating components of DRZ (Figure 1).
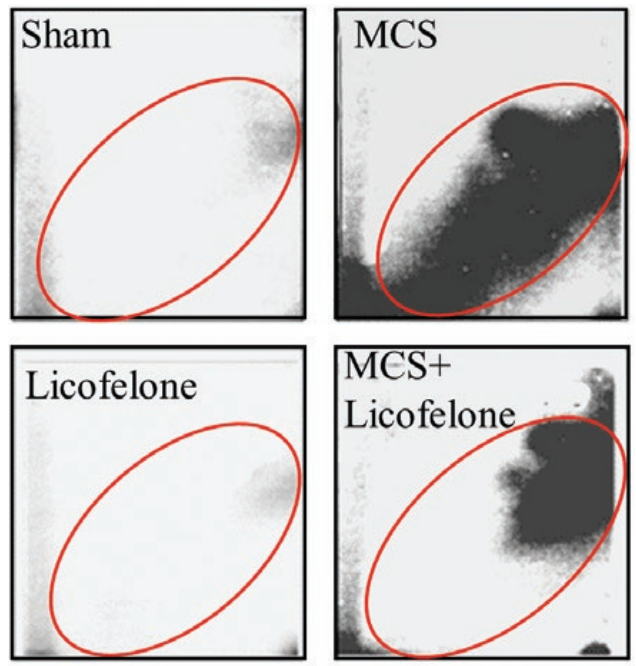

Figure 1. Examples of ${ }^{32} \mathrm{P}$ autoradiographs obtained by testing the lung DNA from mice, either unexposed (Sham) or exposed to MCS and treated with licofelone. The framed areas correspond to the DRZ in MCS-exposed mice where the radioactive signal was measured (see the results in Table 1).
Licofelone inhibits the MCS-induced oxidative DNA damage in lung

As shown in Table 1 (right column), exposure of mice to MCS caused oxidative DNA damage in the lung, with a 3.0-fold increase of 8-oxo-dGuo in males and a 2.8 -fold increase in females. Administration of licofelone, which per se did not affect 8-oxo-dGuo levels in smoke-free mice, significantly attenuated the MCS-related oxidative DNA damage, with a 3.0-fold decrease in males and a 2.8-fold decrease in females. Thus, the values recorded in MCS-exposed mice treated with licofelone were comparable to the 8-oxo-dGuo levels observed in sham-exposed mice.

\section{Licofelone causes a late increase of systemic genotoxic damage in MCS-exposed mice}

The frequency of MN NCE was evaluated in the peripheral blood of 60 mice aged 4 months, immediately after discontinuation of exposure to MCS and after 3 months of administration of licofelone. These mice were a subset of the mice used in a parallel cancer prevention study (19). As shown in Table 2, exposure to MCS of both male and female mice resulted in a small but significant increase in MN NCE frequency, as compared with shamexposed mice. Administration of licofelone to MCS-exposed mice caused a further significant increase in MN NCE frequency in both males and females, when compared with MCS-exposed mice in the absence of chemopreventive agent.

\section{Licofelone dysregulates a number of pulmonary miRNAs while counteracting MCS-related alterations}

Initially, we performed separate analyses of microarray data for males and females (data not shown). Since no gender differences were detected, we decided to merge the results for males and females in order to analyze larger numbers of mice per group. HCA (Figure 2A) showed that Sham and MCS are located in opposite branches of the dendrogram. Licofelone alone is linked with Sham, and MCS + licofelone has an intermediate position, being located in a different branch compared with MCS in the absence of drug. In bidimensional PCA (Figure 2B), licofelone and Sham fell in the same quadrant, whereas MCS + licofelone and MCS were together in another quadrant. However, MCS + licofelone tended to approach the quadrant where Sham and licofelone were allocated. SPAs compared the miRNA expression in licofelone-treated mice versus sham-exposed mice (Figure 3A), MCS-exposed mice versus sham-exposed mice (Figure 3B), and MCS-exposed mice, in the absence of the drug, versus MCSexposed mice treated with licofelone (Figure $3 \mathrm{C}$ ). The symbols falling outside the diagonal belts refer to those miRNAs that varied at least 2-fold, either in the sense of upregulation or of downregulation, in the compared groups.

Volcano-plot analyses confirmed that 40 miRNAs (3.5\%) changed their expression more than 2-fold and above the statistical significance threshold $(P<0.05)$ in smoke-free mice treated with licofelone (Figure 3D). Twenty-six of them were upregulated and 14 were downregulated by the drug. A list of the licofelonedysregulated miRNAs, along with the fold-variation compared with sham-exposed mice, the statistical significance $P$ value, and their main function(s), is reported in Supplementary Table S2, available at Carcinogenesis Online.

As shown in Figure 3B, exposure to MCS, in the absence of licofelone, mainly affected miRNAs expressed at high levels of intensity (upper right part of the expression cloud, red color dots). Volcano-plot analyses (Figure 3E) revealed that 62 miRNAs out of the 1135 tested (5.5\%) were significantly downregulated 
Table 1. Bulky DNA adducts and 8-oxo-dGuo levels in the lung of 40 mice aged 10 weeks as related to gender, exposure to MCS since birth and/ or treatment with licofelone since weaning.

\begin{tabular}{llll}
\hline Treatment & Gender & $\begin{array}{l}\text { DNA adducts/ } \\
10^{8} \text { nucleotides }\end{array}$ & $\begin{array}{l}\text { 8-oxo-dGuo } \\
\text { /105nucleotides }\end{array}$ \\
\hline Sham & Males & $1.31 \pm 0.17$ & $1.70 \pm 0.22$ \\
& Females & $1.23 \pm 0.15$ & $2.11 \pm 0.18$ \\
Licofelone & Males & $0.93 \pm 0.17$ & $1.90 \pm 0.27$ \\
MCS & Females & $1.13 \pm 0.18$ & $2.06 \pm 0.26$ \\
& Males & $17.92 \pm 1.77^{\mathrm{a}}$ & $5.03 \pm 0.08^{\mathrm{a}}$ \\
MCS + licofelone & Females & $20.04 \pm 2.95^{\mathrm{a}}$ & $5.97 \pm 0.44^{\mathrm{a}}$ \\
& Males & $8.40 \pm 0.79^{\mathrm{a}, \mathrm{c}}$ & $1.67 \pm 0.07^{\mathrm{d}}$ \\
\hline
\end{tabular}

The results are means $\pm \mathrm{SE}$ within each group of 5 mice. Statistical analysis: ap $<0.001$, as compared with sham-exposed mice of the same gender; ${ }^{\mathrm{P}}<<0.05$, ${ }^{\mathrm{c}}<<0.01$, and ${ }^{\mathrm{P}} \mathrm{P}<0.001$, as compared with MCS-exposed mice of the same gender.

Table 2. Frequency of MN NCE in the peripheral blood of 60 Swiss $H$ mice aged 4 months, immediately after discontinuation of exposure to MCS and after 3 months of treatment with licofelone.

\begin{tabular}{llll}
\hline Treatment & $\begin{array}{l}\text { Dose of } \\
\text { licofelone } \\
(\mathrm{mg} / \mathrm{kg} \text { diet })\end{array}$ & Gender & $\begin{array}{l}\text { MN NCE (\%) } \\
\text { Mean } \pm \text { SE }\end{array}$ \\
\hline Sham & 0 & M & $1.33 \pm 0.098$ \\
MCS & 0 & F & $0.86 \pm 0.082$ \\
MCS + & 0 & M & $1.93 \pm 0.139^{\mathrm{a}}$ \\
Licofelone & 960 & F & $1.18 \pm 0.059^{\mathrm{a}}$ \\
\hline
\end{tabular}

Each experimental group was composed of 10 mice/gender; 50,000 NCE were examined in each mouse. Statistical analysis: ${ }^{\mathrm{a}} \mathrm{P}<0.01$ and ${ }^{\mathrm{b}} \mathrm{P}<0.001$, as compared with sham-exposed mice of the same gender. ${ }^{\mathrm{c}}<0.01$, as compared with MCS-exposed mice of the same gender, in the absence of licofelone.

by MCS (Supplementary Table S3 is available at Carcinogenesis Online). Licofelone was poorly effective in changing miRNA expression as compared with MCS alone (Figure 3C). In particular, volcano-plot analyses (Figure 3F) showed that licofelone was able to restore the expression of 7 of the 62 MCS-dysregulated miRNAs (11.3\%). A list of these miRNAs, all of which were upregulated by licofelone in MCS-exposed mice, is shown in Supplementary Table S4, available at Carcinogenesis Online.

Validation of microarray data by qPCR for miR-30b (Figure 4) confirmed that this miRNA was upregulated by licofelone when compared with sham (Licofelone/Sham fold variation of 1.7 by qPCR and 3.1 by microarray). Licofelone was effective in contrasting the downregulation of this miRNA in MCS-exposed mice by normalizing its expression (MCS + Licofelone/Sham fold variation of 1.1 by qPCR and 1.3 by microarray).

\section{Discussion}

The results of the present study provide evidence that licofelone succeeded in attenuating the formation of bulky DNA adducts and oxidative DNA damage in the lung of MCS-exposed mice. In addition, the drug was able to modulate the expression of several miRNAs in the lung of mice, either smoke-free or exposed to MCS. Since the mice were exposed to MCS short after birth and received dietary licofelone for 6 weeks after weaning, the protocol that we used mimics a subchronic pharmacological treatment in current smokers. The 10-week period of exposure of mice to MCS covered the immediate post-natal time, during which the sudden transition from the maternal-mediated respiration to the autonomous pulmonary respiration causes paraphysiological genomic alterations in the lung (35), followed by the lactation period until completion of weaning. This period partially overlaps with adolescence, which in mice has been divided into 3 ages, including pre-adolescence (23-35 days), midadolescence (36-48 days) and post-adolescence (49-61 days) (36), leading to sexual maturity and early adulthood. Mice are considered to be adults after 2 months ( 8.6 weeks). The administered dose of licofelone ( $960 \mathrm{mg} / \mathrm{kg}$ diet) was rather high, but it did neither affect survival nor body weight gain in smoke-free mice, which is in agreement with the results of a preliminary 6 -week subchronic toxicity study using post-weanling mice under the same experimental conditions (19). Even more, in the present study, licofelone exerted some protective effects against the small but significant loss of body weight gain induced by MCS 3-6 weeks after weanling. It should be also taken in mind that, while licofelone is administered once or twice per day in humans, the intake of the drug in mice with the diet is highly fractionated because rodents eat continuously depending on their metabolic needs, which is expected to result in a greater tolerability. However, as shown in the part of the present study evaluating modulation of systemic genotoxicity and in the parallel cancer chemoprevention study (19), licofelone exhibited some signs of toxicity, in terms of a decrease in survival and body weight gain, when it was administered for more than 4 months to MCS-exposed mice.

In agreement with the results of previous studies (21,22,3741), exposure of mice to MCS resulted in an evident oxidative DNA damage in lung. Moreover, high levels of bulky DNA adducts were detectable in the form of DRZ at ${ }^{32} \mathrm{P}$ postlabeling, which is typical for exposure to complex mixtures. MCSinduced DNA alterations were of the same order of magnitude in male and female mice. As shown in mice exposed to CS, oxidative stress and the resulting DNA damage provide a major contribution to the high susceptibility of mice exposed early in life (42). Administration of licofelone to MCS-exposed mice approximately halved DNA adduct levels in both genders by mainly inhibiting slowly migrating components of DRZ. These include lipophilic bulky adducts resulting from DNA adduction with polycyclic aromatic hydrocarbons and lipid peroxide metabolites. Even greater (about three times) was inhibition of oxidative DNA damage in the lung of MCS-exposed mice, to such an extent that 8-oxo-dGuo levels in MCS-exposed mice treated with licofelone were reverted to the same levels observed in sham-exposed mice.

On the other hand, administration of licofelone caused a slight but statistically significant increase in MN NCE frequency 

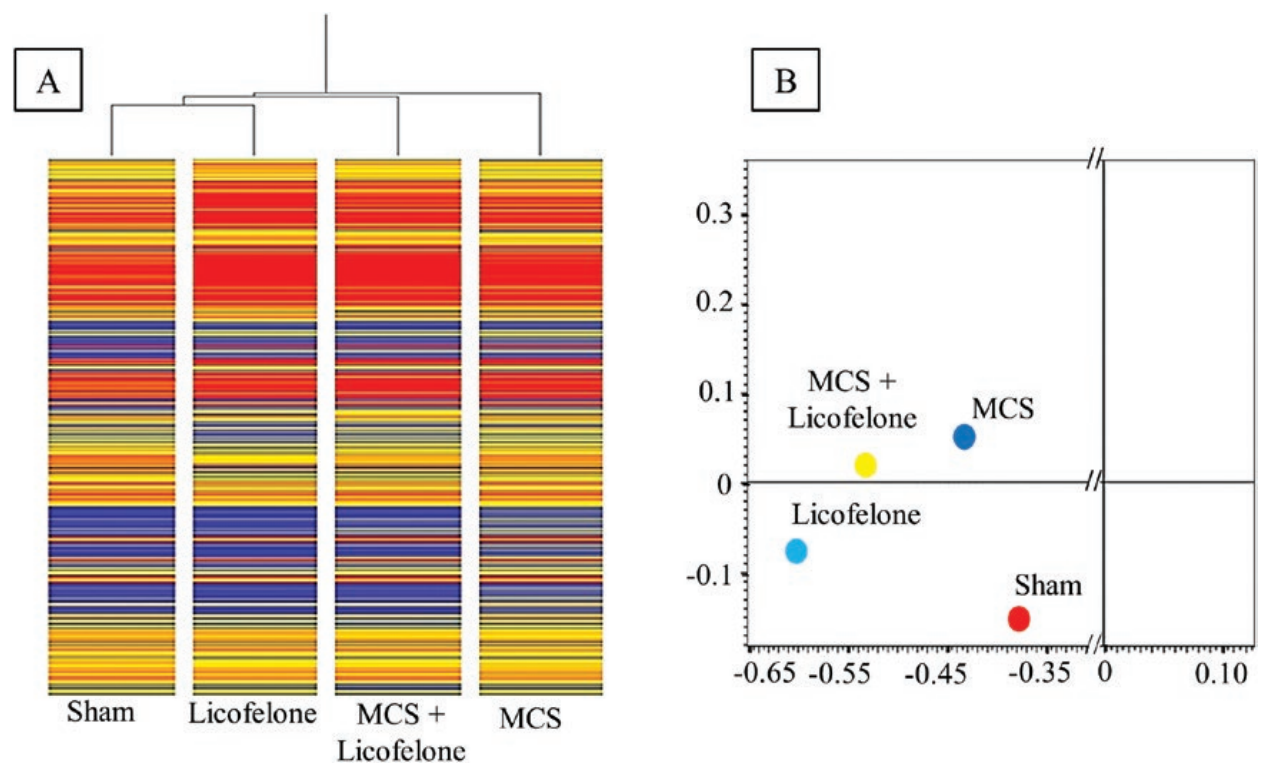

Figure 2. Global expression of 1135 pulmonary miRNAs in the mice belonging to 4 experimental groups, including sham-exposed mice, mice receiving licofelone with the diet for 6 weeks, starting after weanling, and mice exposed to MCS for 10 weeks, starting after birth, either untreated or treated with licofelone. (A) Hierarchical cluster analysis (HCA). Within each column, the expression of the analyzed lung miRNAs is represented on a relative color scale (blue, low; yellow, medium; orange to red, high). (B) Bidimensional principal component analysis (PCA) showing the allocation of the average expression of pulmonary miRNAs in the mice belonging to four experimental groups.
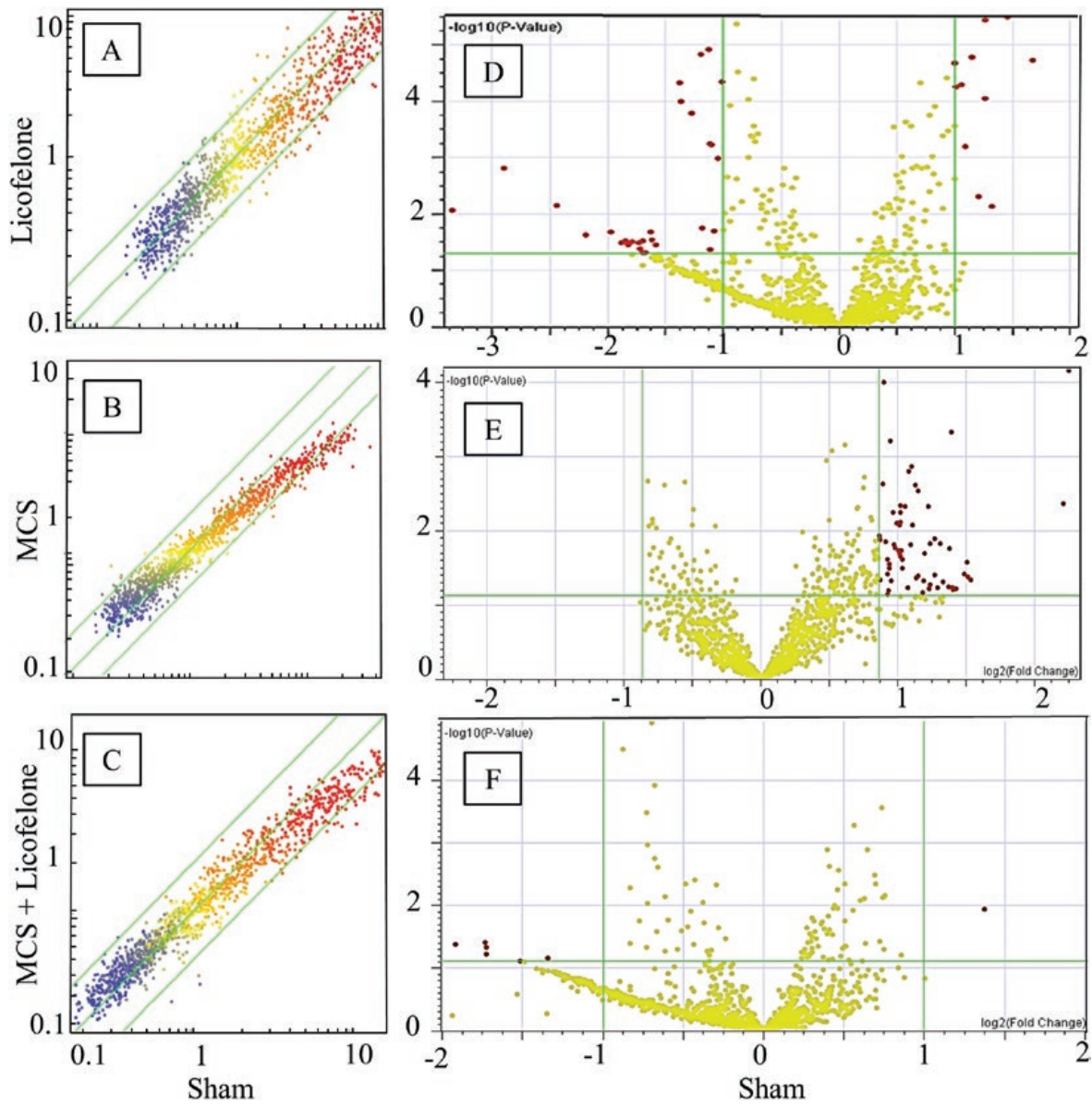

Figure 3. (A-C) Scatter-plot analyses comparing the expression of pulmonary miRNAs in sham-exposed mice us. mice treated with licofelone (A), MCS-exposed mice us. sham-exposed mice (B), and MCS-exposed mice treated with licofelone versus MCS-exposed mice in the absence of the drug (C). Each dot represents a miRNA, whose expression intensity can be inferred from the position in the $x$ and $y$ axes, according to a color scale (blue, low; yellow, medium; orange to red, high). The diagonal belts indicate the 2-fold variation interval. Symbols falling in the upper area denote miRNA upregulation, and those falling in the lower area denote miRNA downregulation by licofelone. (D-F) Volcano-plot analyses comparing the expression of pulmonary miRNAs in sham-exposed mice versus mice treated with licofelone (D), MCS-exposed mice versus sham-exposed mice (E), and MCS-exposed mice treated with licofelone versus MCS-exposed mice in the absence of the drug ( $F$ ). The two green vertical lines indicate the 2-fold variation interval, and the horizontal green line indicates the $P<0.05$ threshold. miRNAs falling outside these intervals are highlighted in red color. 


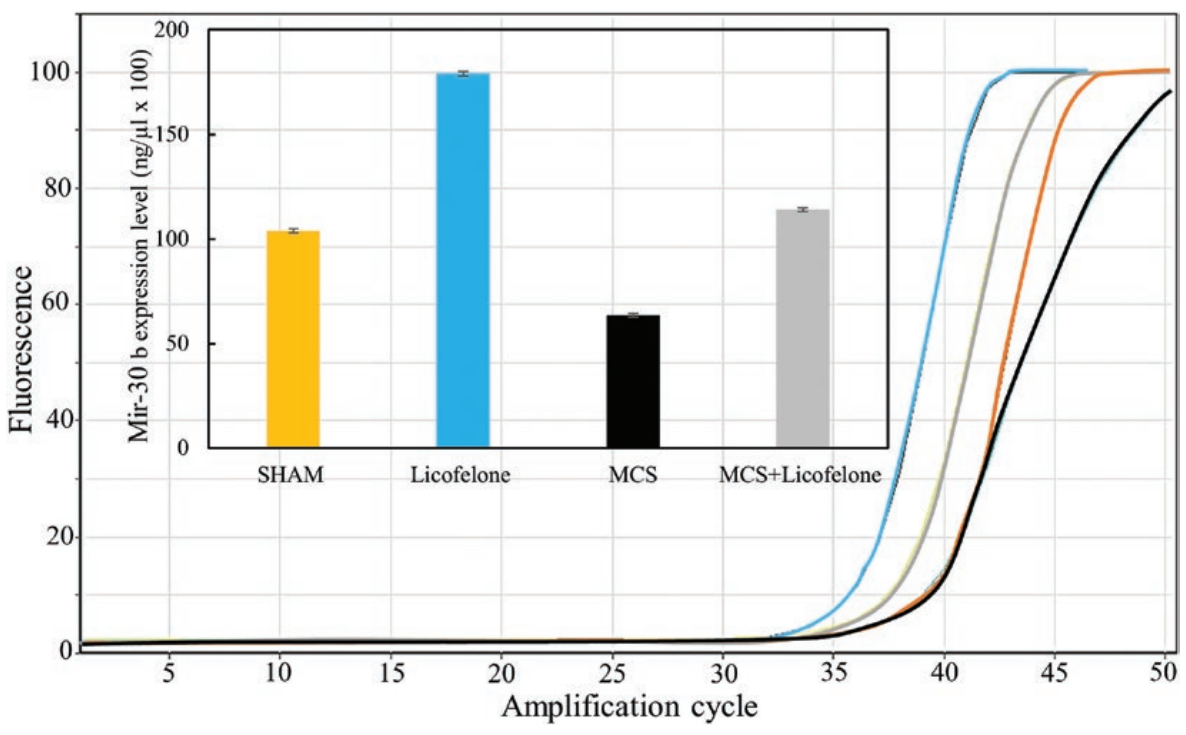

Figure 4. Evaluation of miR-30b expression by qPCR. Amplification curves are reported for each one of the four experimental conditions. The column graph quantifies the level of miR-30b expression.

in the mice aged 4 months, as compared with MCS-exposed mice in the absence of chemopreventive agent. This finding was unexpected but consistent in both males and females. At that time, as previously mentioned, MCS-exposed mice treated with licofelone underwent a further decrease in survival and body weight gain as compared with mice exposed to MCS in the absence of chemopreventive treatment. Therefore, such an enhancement of systemic genotoxicity may reflect some toxicity of the drug after a longer administration to MCS-exposed mice. Targeting upstream enzymes affects multiple downstream pathways and may produce adverse effects by disrupting the balance between different AA-metabolizing pathways (43). Presumably, the simultaneous inhibition by licofelone of the COX-1/2 and 5-LOX pathways may shunt the metabolism of AA via the CYP pathway thereby favoring the formation of free radical products and modulating the metabolism of carcinogens contained in MCS (44).

Even after 6 weeks only, administration of licofelone to smoke-free mice caused an extensive dysregulation of pulmonary miRNAs, which may underlie the occurrence of either protective mechanisms and/or possible alterations of the physiological homeostasis. In particular, out of 40 miRNAs dysregulated by licofelone, 26 were upregulated and 14 were downregulated. The emerging picture is rather intricate, also because miRNAs possess pleiomorphic properties and regulate a number of genes simultaneously, a single miRNA targeting approximately 200 transcripts (45). The miRNAs modulated by dietary licofelone in mouse lung included miRNAs that are mainly involved in inflammation (miR-125b, miR-146a, miR-147, miR-149, miR-574), stress response (miR-30b, miR-146a, miR342), cell proliferation (let-7f, miR-1a, miR-362, miR-466h, miR574, miR-1224, miR-3064), apoptosis (miR-1a, miR-98, miR-149, miR-204, miR-574, miR-700), cell adhesion (let-7c, miR-30b, miR135b, miR-205, miR-592), cell differentiation (miR-143, miR-204, miR-363, miR-705), angiogenesis (let-7f, miR-98, miR-205, miR592), regulation of $\mathrm{k}$-Ras (let-7f, miR-135b, miR-143, miR-466h) or other oncogenes (miR-98, miR-125b, miR-149, miR-381, miR542, miR-744), and modulation of suppressor genes (miR-190a, miR-1224)

Two miRNAs involved in cardiovascular development (miR3065) and in the regulation of cardiac hyperthrophy (miR-672) were also upregulated by licofelone. miR-672 showed suppressive effects on cardiac hypertrophy through regulating the expression of Jun in cardiomyocytes (46). As mentioned in the Introduction, targeting both COX and 5-LOX pathways may avoid cardiovascular side effects (17), and 5-LOX has been identified as a major gene contributing to atherosclerosis susceptibility in mice (47). The only miRNA upregulated by licofelone that is involved in COX-2 regulation was miR-146a. This miRNA is downregulated in lung cancer cells thereby increasing COX-2 expression and the related inflammation (48). The fact that one only of the 40 licofelone-modulated miRNAs targeted COX-2 suggests that COX-2 inhibition by licofelone is exerted by direct interaction with the enzyme and not by epigenetic modulation.

In line with previous studies (22,39-41,49-55), exposure of mice to MCS resulted in an extensive dysregulation of pulmonary miRNAs, which was oriented in the sense of downregulation. The reliability of microarrays for evaluating miRNA dysregulation in the lung of MCS-exposed mice was validated by means of qPCR analyses in the present study and in several previous studies investigating the effects of MCS and other putative chemopreventive agents (vorinostat, myoinositol, bexarotene, pioglitazone, metformin, lapatinib, aspirin, naproxen, and celecoxib) under experimental conditions similar to those used in the present study $(22,39-41,49,54,55)$. The 62 miRNAs downregulated by MCS in the present study are involved in a variety of functions, mainly including oncogene inhibition, stress response, apoptosis and angiogenesis. These findings underlie the fact that epigenomic alterations overwhelm genomic alterations in MCS-related pulmonary carcinogenesis. Indeed, in a separate study we explored oncogene k-ras hotspot mutations by pyrosequencing in the lung of cancer-free mice, either sham-exposed or exposed to MCS for 10 weeks. As assessed in two separate laboratories, no mutation $(\mathrm{G} \rightarrow \mathrm{T}$ transversion, codon $12 / 13$ ) was detected in any of the 20 mice examined (Izzotti et al., manuscript in preparation). The observed lack of mutation of k-ras in MCS-exposed mice is consistent with Cosmic data (https://cancer.sanger.ac.uk/cosmic) reporting that, in human full blown lung cancer, k-ras mutation frequency is $16 \%$ only.

In MCS-exposed mice, licofelone upregulated 7 miRNAs, thus restoring their expression that had been downregulated 
by MCS exposure. The expression of 3 of these miRNAs (miR$30 \mathrm{~b}, \mathrm{miR}-125 \mathrm{~b}, \mathrm{miR}-466 \mathrm{~h}$ ) was also upregulated by licofelone in smoke-free mice. Interestingly, a homologue of miR-30b (miR30e) and two homologues of miR-466h (miR-466a and miR-466i) had been found to be significantly less expressed in the lungs of adenoma-bearing as compared with adenoma-free 7.5-monthold Swiss albino mice exposed to MCS during the first 4 months of life and treated with the nonselective COX-1/COX-2 inhibitor aspirin after weaning (22). These findings suggest that these miRNAs play a protective role in MCS-related carcinogenesis since they were more abundant in the lung of adenoma-free mice than in the lung of adenoma-bearing mice sharing exposure to MCS. A similar situation occurred in mice treated with the other nonselective NSAID naproxen, in which another miR-30b homologue (miR-30c) was significantly more expressed in MCS-exposed mice treated with the drug that were free of adenomas than in those bearing adenomas (22). On the other hand, the miRNAs upregulated by the selective COX-2 inhibitor celecoxib in the lung of MCS-exposed Swiss H mice were different from those upregulated by licofelone under identical experimental conditions (21). As to miR-125b, this miRNA having multiple functions was upregulated both in the lung of Swiss $\mathrm{H}$ mice exposed for 10 weeks to MCS since birth and treated with licofelone after weaning (present study) and in the lung of 2-month old ICR(CD-1) mice exposed to MCS for 8 weeks and receiving celecoxib during the same period (21).

The experimental model used in the present study is suitable to evaluate the protective effects of a putative chemopreventive agent towards MCS, which is the most important human carcinogen. At the same time, such a methodological approach is also suitable to evaluate possible adverse effects because the drug is tested not only in in MCS-exposed mice but also in smoke-free mice. In principle, the ability of a given agent to counteract the DNA and RNA alterations induced by MCS may be assumed as a molecular indicator of efficacy, whereas a poor disturbance of DNA and RNA physiological expressions in smoke-free mice may be assumed as a molecular indicator of safety.

In this light, licofelone exerted convincing protective effects towards MCS but, at the same dose, it produced some interference with the physiological molecular machinery in smoke-free mice. Both trends correlated with the modulation of pathological alterations resulting from exposure to MCS and/or treatment with this NSAID. In particular, at a dose and treatment time (6 weeks) that did not produce any sufferance or body loss in mice but, on the contrary, it protected the mice from the MCSinduced body weight loss, dietary licofelone caused an early inhibition of oxidative DNA damage and bulky DNA adducts induced by MCS in mouse lung. This was accompanied by modulation of the expression of miRNAs involved in inflammation, stress response and other protective functions. Such an outcome is consistent with the known anti-inflammatory properties of this NSAIDS and correlates with the finding that, in the medium term (7.5 months), licofelone protected the mice from MCSinduced pulmonary emphysema, bronchial epithelial hyperplasia, and it attenuated the formation of lung microadenomas, which are probably related to MCS-related chronic inflammation (19). Microadenomas are larger than hyperplastic foci, tend to regress spontaneously (56), and do not necessarily contribute to the development of lung tumors (40). In addition, consistently with the notions that chronic inflammation plays a key role in all stages of the carcinogenesis process (8) and that the COX-2/PGE2 signaling pathway is involved in the development of malignant cells (57), the yield of malignant tumors in the lungs of MCS-exposed mice treated with licofelone was lower when compared with MCS-exposed mice that did not receive the drug (19). This finding is in agreement with the conclusions of another study, in which licofelone administration attenuated the benzo(a)pyrene-induced yield of lung tumors in mice after that dysplasia had already developed (28). In the direct comparison of licofelone with celecoxib, the former NSAIDS showed in general better protective effects (40), consistently with the assumption that triple inhibitors that block COX-1, COX-2 and 5-LOX may provide synergistic anti-inflammatory effects compared with COX inhibitors (23). On the other hand, administration of licofelone to smoke-free mice, even in the short term, resulted in a broad dysregulation of pulmonary miRNAs, some of which may trigger adverse effects. In addition, after 4 months licofelone slightly but significantly enhanced the MCS-related systemic genotoxicity (present study) and, after that time, administration of licofelone to MCS-exposed mice worsened survival and body weight gain (19).

In conclusion, the results of the present study provide evidence that evaluation of early genomic and epigenetic endpoints highlights the ability of licofelone to counteract certain mechanisms involved in the pathogenesis of cancer and other chronic degenerative diseases, thus explaining the previously observed cancer protective effects. In the long term, however, the licofelone dose used also exhibited some toxic effects. Therefore, caution should be paid when administering licofelone to smokers.

\section{Supplementary material}

Supplementary data are available at Carcinogenesis online.

\section{Funding}

This work was supported by the US National Cancer Institute (HHSN-261200433000C to S.D.F.), by the Italian Association for Cancer Research (AIRC, IG2017-20699 to A.I.) and by the Bulgarian Ministry of Science - National Science Fund (BIN-1 to R.B).

Conflict of Interest Statement: None declared.

\section{References}

1. International Agency for Research on Cancer. (2012). A review of human carcinogens: personal habits and indoor combustions. IARC Monographs on the Evaluation of the Carcinogenic Risks to Humans, Vol. 100E. IARC, Lyon, France.

2. De Flora, S. et al. (2014) Rationale and approaches to the prevention of smoking-related diseases: overview of recent studies on chemoprevention of smoking-induced tumors in rodent models. J. Environ. Sci. Health. C. Environ. Carcinog. Ecotoxicol. Rev., 32, 105-120.

3. Hecht, S.S. et al. (2014) Fifty years of tobacco carcinogenesis research: from mechanisms to early detection and prevention of lung cancer. Cancer Prev. Res. (Phila)., 7, 1-8.

4. De Flora, S. et al. (2005) Overview of mechanisms of cancer chemopreventive agents. Mutat. Res., 591, 8-15.

5. American Cancer Society (2018). Cancer Facts \& Figures 2018. ACS, Atlanta, GA.

6. Weiss, W. (1997) Cigarette smoking and lung cancer trends. A light at the end of the tunnel? Chest, 111, 1414-1416.

7. Malkinson, A.M. (2004) Evidence that inflammation encourages pulmonary adenocarcinoma formation in mice: clinical implications. Chest, 125 (5 Suppl), 154S-155S.

8. Grivennikov, S.I. et al. (2010) Immunity, inflammation, and cancer. Cell, 140, 883-899.

9. Takahashi, H. et al. (2010) Tobacco smoke promotes lung tumorigenesis by triggering IKKbeta- and JNK1-dependent inflammation. Cancer Cell, 17, 89-97. 
10. Smith, C.J. et al. (2006) Perspectives on pulmonary inflammation and lung cancer risk in cigarette smokers. Inhal. Toxicol., 18, 667-677.

11. Aggarwal, B.B. et al. (2006) Inflammation and cancer: how hot is the link? Biochem. Pharmacol., 72, 1605-1621.

12. Harris, R.E. (2007) Cyclooxygenase-2 (cox-2) and the inflammogenesis of cancer. Subcell. Biochem., 42, 93-126.

13. Lee, I.T. et al. (2013) Inflammatory signalings involved in airway and pulmonary diseases. Mediators Inflamm., 2013, 791231.

14. Mazhar, D. et al. (2005) COX and cancer. QJM., 98, 711-718.

15. Wolfe, F. et al. (2002) Gastroprotective therapy and risk of gastrointestinal ulcers: risk reduction by COX-2 therapy. J. Rheumatol., 29, 467-473.

16. Duffield-Lillico, A.J. et al. (2009) Levels of prostaglandin E metabolite and leukotriene $\mathrm{E}(4)$ are increased in the urine of smokers: evidence that celecoxib shunts arachidonic acid into the 5-lipoxygenase pathway. Cancer Prev. Res. (Phila)., 2, 322-329.

17. Mohammed, A. et al. (2011) Chemoprevention of colon and small intestinal tumorigenesis in APC(Min/+) mice by licofelone, a novel dual 5-LOX/COX inhibitor: potential implications for human colon cancer prevention. Cancer Prev. Res. (Phila)., 4, 2015-2026.

18. De Flora, S. et al. (2016) Pharmacological modulation of lung carcinogenesis in smokers: preclinical and clinical evidence. Trends Pharmacol. Sci., 37, 120-142.

19. Balansky, R. et al. (2015) Modulation by licofelone and celecoxib of experimentally induced cancer and preneoplastic lesions in mice exposed to cigarette smoke. Curr. Cancer Drug Targets., 15, 188-195.

20. Balansky, R. et al. (2016) Selective inhibition by aspirin and naproxen of mainstream cigarette smoke-induced genotoxicity and lung tumors in female mice. Arch. Toxicol., 90, 1251-1260.

21. Izzotti, A. et al. (2018) Modulation of genomic and epigenetic endpoints by celecoxib. Oncotarget, 9, 33656-33681.

22. Izzotti, A. et al. (2017) Early and late effects of aspirin and naproxen on microRNAs in the lung and blood of mice, either unexposed or exposed to cigarette smoke. Oncotarget, 8, 85716-85748.

23. Fiorucci, S. et al. (2001) Dual inhibitors of cyclooxygenase and 5-lipoxygenase. A new avenue in anti-inflammatory therapy? Biochem. Pharmacol., 62, 1433-1438.

24. Alvaro-Gracia, J.M. (2004) Licofelone-clinical update on a novel LOX/ COX inhibitor for the treatment of osteoarthritis. Rheumatology (Oxford)., 43 (Suppl 1), i21-i25.

25. Mohammed, A. et al. (2018) Clinically relevant anti-inflammatory agents for chemoprevention of colorectal cancer: new perspectives. Int. J. Mol. Sci., 19, pii:E2332.

26. Madka, V. et al. (2014) Chemoprevention of urothelial cell carcinoma growth and invasion by the dual COX-LOX inhibitor licofelone in UPIISV40T transgenic mice. Cancer Prev. Res. (Phila)., 7, 708-716.

27. McCormick, D.L. et al. (2012) Chemoprevention of rat oral carcinogenesis by licofelone, a dual cyclooxygenase/lipoxygenase inhibitor. Cancer Prev. Res. (Phila)., 5, B110.

28. Sharma, S. et al. (2011) Chemopreventive efficacy and mechanism of licofelone in a mouse lung tumor model via aspiration. Cancer Prev. Res. (Phila)., 4, 1233-1242.

29. Rao, C.V. et al. (2015) Simultaneous targeting of 5-LOX-COX and EGFR blocks progression of pancreatic ductal adenocarcinoma. Oncotarget, 6 , 33290-33305.

30. Mohammed, A. et al. (2015) Targeting pancreatitis blocks tumorinitiating stem cells and pancreatic cancer progression. Oncotarget, 6 , 15524-15539.

31. Kumar, G. et al. (2016) Simultaneous targeting of 5-LOX-COX and ODC block NNK-induced lung adenoma progression to adenocarcinoma in A/J mice. Am. J. Cancer Res., 6, 894-909.

32. Balansky, R. et al. (2007) Potent carcinogenicity of cigarette smoke in mice exposed early in life. Carcinogenesis, 28, 2236-2243.

33. Izzotti, A. et al. (1999) Formation and persistence of nucleotide alterations in rats exposed whole-body to environmental cigarette smoke. Carcinogenesis, 20, 1499-1505.
34. Izzotti, A. et al. (2001) Modulation of biomarkers by chemopreventive agents in smoke-exposed rats. Cancer Res., 61, 2472-2479.

35. Izzotti, A. et al. (2003) Birth-related genomic and transcriptional changes in mouse lung. Modulation by transplacental N-acetylcysteine. Mutat. Res., 544, 441-449.

36. Adriani, W. et al. (2004) Behavioral and neurochemical vulnerability during adolescence in mice: studies with nicotine. Neuropsychopharmacology, 29, 869-878.

37. De Flora, S. et al. (2003) Modulation of cigarette smoke-related endpoints in mutagenesis and carcinogenesis. Mutat. Res., 523-524, 237-252.

38. De Flora, S. et al. (2005) Induction and modulation of lung tumors: genomic and transcriptional alterations in cigarette smoke-exposed mice. Exp. Lung Res., 31, 19-35.

39. Izzotti, A. et al. (2014) Modulation by metformin of molecular and histopathological alterations in the lung of cigarette smoke-exposed mice. Cancer Med., 3, 719-730.

40. Balansky, R. et al. (2014) Assay of lapatinib in murine models of cigarette smoke carcinogenesis. Carcinogenesis, 35, 2300-2307.

41. Izzotti, A. et al. (2018) Release of microRNAs into body fluids from ten organs of mice exposed to cigarette smoke. Theranostics, 8, 2147-2160.

42. Micale, R.T. et al. (2013) Oxidative stress in the lung of mice exposed to cigarette smoke either early in life or in adulthood. Arch. Toxicol., 87, 915-918.

43. Chen, X. et al. (2006). Five-lipoxygenase pathway of arachidonic acid metabolism in carcinogenesis and cancer chemoprevention. Curr. Cancer Drug Targets., 6, 613-622.

44. Anttila, S. et al. (2011) Cytochrome P450-mediated pulmonary metabolism of carcinogens: regulation and cross-talk in lung carcinogenesis. Am. J. Respir. Cell Mol. Biol., 44, 583-590.

45. Brennecke, J. et al. (2005) Principles of microRNA-target recognition. PLoS Biol., 3, e85.

46. Lu, Y. et al. (2018) A new miRNA regulator, miR-672, reduces cardiac hypertrophy by inhibiting JUN expression. Gene, 648, 21-30.

47. Mehrabian, M. et al. (2002) Identification of 5-lipoxygenase as a major gene contributing to atherosclerosis susceptibility in mice. Circ. Res., 91, 120-126.

48. Cornett, A.L. et al. (2014) Regulation of COX-2 expression by miR-146a in lung cancer cells. RNA, 20, 1419-1430.

49. Izzotti, A. et al. (2009) Relationships of microRNA expression in mouse lung with age and exposure to cigarette smoke and light. FASEB J., 23, 3243-3250.

50. Izzotti, A. et al. (2010) Modulation of microRNA expression by budesonide, phenethyl isothiocyanate and cigarette smoke in mouse liver and lung. Carcinogenesis, 31, 894-901.

51. Izzotti, A. et al. (2011) Interplay between histopathological alterations, cigarette smoke and chemopreventive agents in defining microRNA profiles in mouse lung. Mutat. Res., 717, 17-24.

52. Izzotti, A. et al. (2011) Dose-responsiveness and persistence of microRNA expression alterations induced by cigarette smoke in mouse lung. Mutat. Res., 717, 9-16.

53. De Flora, S. et al. (2012) Smoke-induced microRNA and related proteome alterations. Modulation by chemopreventive agents. Int. J. Cancer., 131, 2763-2773.

54. Izzotti, A. et al. (2013) Relationships between pulmonary micro-RNA and proteome profiles, systemic cytogenetic damage and lung tumors in cigarette smoke-exposed mice treated with chemopreventive agents. Carcinogenesis, 34, 2322-2329.

55. Izzotti, A. et al. (2016) Blood and lung microRNAs as biomarkers of pulmonary tumorigenesis in cigarette smoke-exposed mice. Oncotarget, 7 , 84758-84774.

56. O'Donnell, E.P. et al. (2006) Quantitative analysis of early chemicallyinduced pulmonary lesions in mice of varying susceptibilities to lung tumorigenesis. Cancer Lett., 241, 197-202.

57. Menter, D.G. et al. (2010) Cyclooxygenase-2 and cancer treatment: understanding the risk should be worth the reward. Clin. Cancer Res., 16, 1384-1390. 